\title{
Telepsychiatry and other cutting edge technologies in Covid-19 pandemic: bridging the distance in mental health assistance
}

\author{
Francesco Di Carlo ${ }^{1}$, Antonella Sociali ${ }^{1}$, Elena Picutti ${ }^{1}$, Mauro Pettorruso ${ }^{1}$, Federica \\ Vellante $^{1}$, Valeria Verrastro ${ }^{1}$, Giovanni Martinotti ${ }^{1}$, and Massimo di Giannantonio ${ }^{1}$ \\ ${ }^{1}$ Affiliation not available
}

May 21, 2020

\begin{abstract}
BACKGROUND: Initially identified in December 2019 in China, the 2019 Coronavirus disease (COVID-19) is now affecting more than 200 countries and territories around the world. Given the current unavailability of an effective medical cure for COVID19, a public health strategy of reduced social contact and shelter in place has been adopted worldwide. Nonetheless, social distancing and isolation could also represent risk factors for mental disorders, determining loneliness, reduced social support and under-detection of mental health needs. Along with this, social distancing determines an insurmountable obstacle for direct access to psychiatric care services. The pandemic generates the urgent need for integrating technology into innovative models of mental healthcare. AIMS: In this paper we discuss the potential role of telepsychiatry and other cutting-edge technologies in the management of mental health assistance. We narratively review the literature to examine advantages and risks related to the massive application of these new therapeutic settings, along with the possible limitations and ethical concerns. RESULTS: Telemental health services are particularly feasible and appropriate for the support of patients, family members and health-care providers during this COVID-19 pandemic. The integration of telepsychiatry with other technological innovations (e.g., mobile apps, virtual reality, big data and artificial intelligence) opens up interesting future perspectives for the improvement of mental health assistance. CONCLUSION: The COVID-19 pandemic situation shows us how vast the amount of untreated mental illnesses can be. The pandemic crisis can contribute to spread, among numerous mental health professionals, the knowledge of the possibilities offered by the digital era.
\end{abstract}

\section{Introduction}

Initially identified in December 2019 in China, the 2019 Coronavirus disease (COVID-19) is now affecting more than 200 countries and territories around the world. Given the current unavailability of an effective medical cure for COVID-19, a public health strategy of reduced social contact and shelter-in-place has been adopted worldwide to slow the spread of the virus. This strategy may on the one hand achieve its goals, but on the other hand, it may undermine normal social support systems, yielding to loneliness and reduced support from family and friends. These conditions may be risk factors for the development of anxiety, stress and depressive symptoms in the general population and they may also worsen or decompensate psychiatric diseases previously diagnosed [1]. The above listed symptoms may lead to long-term consequences on the patients' health, especially if not properly treated. Since physical access to the Mental Health Care System increases the risk of infection, telemental health services can be particularly useful and appropriate for the support of both patients and health care providers during this pandemic. In fact, technology-based tools offer telemedicine visits for patients, as well as support for institutions, by facilitating the provision of information and data sharing, while creating virtual spaces for meetings and clinical briefings [2].

According to the World Health Organization, telemedicine is "the delivery of health care services, where distance is a critical factor, by all health care professionals, using information and communication technologies for the exchange of valid information for diagnosis, treatment and prevention of diseases and injuries, research 
and evaluation and for the continuing education of health care providers, all in the interests of advancing the health of individuals and their communities" [3]. When applied in the field of psychiatry these methods are known as telepsychiatry (TP). It is interesting to underline that psychiatric care is relatively unique: with only rare exceptions, psychiatrists are not expected to physically touch their patients to provide the best services. Verbal and visual assessments, which can easily occur via telemedicine, are all that is needed to engage with patients appropriately, obtain full diagnostic accuracy and provide most types of treatments. This makes using a 2-way tele-videoconferencing for clinical assessments and treatment delivery a reasonable method for psychiatric treatment [4].

Technologies used in TP can be divided into synchronous and asynchronous. Synchronous technologies include real-time videoconferencing, phone conversations and real-time chat. Provision of mental health services through synchronous communication simulates interviews and face-to-face treatments, although its efficiency is susceptible to the quality of the technology used. Technical complications have recently become less problematic thanks to the development of high-quality video transmission platforms and appropriate confidentiality and security conditions, like Zoom , Blue- jeans, Doxy.me, thera-LINK, TheraNest,SimplePractice, and Vsee [5].

Asynchronous technologies, which provide an alternative to traditional synchronous technologies, can help patients and clinicians communicate without the need for the consultation to occur simultaneously. Some examples of asynchronous TP technologies are: e-mail, SMS text messaging, asynchronous videos, computerized guided therapy, interactive computer programs, mobile device sensors and mobile app-based psychotherapy and psychoeducation [6].

Although for the most severe patients in-person visits are still necessary, TP can be considered a valuable way of supporting psychological and social needs of individuals, regardless of their geographical location.

The aim of this narrative review is to comprehensively update and summarize the most important findings about the use of TP in the modern psychiatric practice, the related strengths, limits, barriers and fields of application.

\section{Methods}

A PubMed search for peer-reviewed original research studies was performed with date limits ranging from January 1st, 1990 to March 15th, 2020. The terms used included: telepsychiatry, telemental health, telehealth, telemedicine, videoconferencing, internet-based, online anddigital. The articles were selected for inclusion by three independent reviewers on the basis of relevance. The reference section of each examined article was also reviewed to identify other potential studies.

\section{Advantages of telepsychiatry}

In their review Cowan et al. [7] reported several advantages to TP, both from the patient's and the clinician's points of view. By working in different settings (schools, prisons, homes, and hospitals) and with different populations (prisoners, students, employees, hospital patients and outpatients) clinicians can have flexibility in scheduling appointments and an increased diversity of practice. They may also feel safer when evaluating patients without any risk of physical aggression. Furthermore, the use of TP allows clinicians to remotely consult other colleagues.

For patients, TP improves access to care, reduces appointments' waiting time and decreases travel time and costs [8]. Some patients report feeling more comfortable, and can be more open and honest, when discussing difficult subjects from their devices, because the virtual space of the session instills a feeling of "protection". Another important advantage of TP in terms of rapport building is the possibility for patients (especially immigrants, refugees, and asylum seekers) to receive psychiatric assistance in their native language without the assistance of an interpreter.

Given a reasonable initial apprehension, discomfort and fear, patients generally report an increase in comfort and satisfaction after having used TP a few times. Comparably to patients, after a few trials, clinicians reveal 
an improved attitude toward TP as well, suggesting that increased exposure for clinicians may be important to alleviate their concerns about rapport.

With regards to efficacy, in a recent review by Hilty et al. [9], TP was found to be effective in terms of patient acceptance, ability to increase access to care and provision of good educational outcomes. Moreover, $\mathrm{TP}$ was found to be valid and reliable when compared to in-person services.

\section{Concerns about telepsychiatry}

Both users and, particularly, nonusers of telemedicine reported disliking the loss of personal contact with patients. The decreased ability to detect nonverbal cues during video conferencing may limit the building of the relationship, since clinicians report some difficulties in picking up nuances and emotions [10]. Some clinicians may feel and look clumsy and uncomfortable, or may have a hard time engaging with patients; eye contact can feel artificial across the screen as well. Clinicians have also expressed discomfort in being unable to take physical actions to reassure or assist their patients. When randomly assigned to evaluate in-person vs videoconference therapy sessions psychiatrists reported lower therapeutic alliance in telemental health settings; they also persisted on being hesitant to use video conference for their therapy sessions because they believed that the therapeutic alliance was at risk. Concerns have been voiced about certain patients possibly being non-suitable for TP, including individuals with psychotic symptoms, acute crisis or at risk of self-harm. Patients with auditive, visual or cognitive impairments appear not to be eligible for telemental health services [7]. Furthermore, it has been proposed to carefully consider the role of substance abuse and past episodes of self-injurious or violent behavior before selecting a patient for a TP intervention [11]. An early recognition of signs of an imminent crisis is essential; in fact, important cues such as facial expressions, tone and posture can be missed in the TP consultation. For this reason, there must be regular screenings for adverse events and a procedure for crisis intervention must be readily available [12]. An important recommendation is to connect with on-site staff who may intervene if necessary [7]. A well-functioning TP system is essential for success. Technical difficulties such as transmission unable to start, spontaneous disconnections, poor audio/visual quality and audio/visual delay may seriously affect the TP session. Sound quality may affect more substantially the interaction; although audio quality may be prioritized, poor visual transmission, resulting in decreased ability to detect nonverbal cues, remains a concern [13]. Finally, clinicians are often reluctant to try TP because it is not something they are accustomed to [14]. New approaches to professional techniques require deliberate conscious efforts. There are deep-rooted cultural traditions in medicine that impede the diffusion of TP. Physical proximity has an important part in clinical practice, with an enormous cultural significance. Over the course of two thousand years, a doctor's physical presence has been regarded as necessary for clinical practice and patients' healing.

\section{Privacy issues}

A relevant concern about using TP is privacy. It is important to ensure conversations' confidentiality and to protect health information and sensitive data. Patients should be educated about personal privacy problems rising from using telemental health services outside the traditional clinical setting, like when speaking in rooms where family members can hear the conversation, or in public places where anyone might listen [8]. The clinician himself must maintain privacy rules and ensure that no one will interrupt the session [11]. Another confidentiality issue is of technological nature, concerning network security and encryption [7]; in fact, videoconferencing occurs through the Internet connection, it is therefore essential to guarantee that the meeting will not be easily hacked into, or viewed by others [4]. Clinicians and organizations who are supplying telemental health services must adapt their connection pathways and equipment to the health information protection laws, such as the USA's Health Insurance Portability and Accountability Act (HIPAA). In recent years a variety of platforms (like Zoom, Bluejeans, SimplePractice and others) have been developed with opportune security and confidentiality [5], but privacy cannot be guaranteed in its entirety. In fact, the FBI warned about the weakness of such platforms after some unidentified users invaded several school-sessions, a phenomenon called "zoombombing". Because of the COVID-19 pandemic, some governments have issued an emergency waiver suspending the requirement to comply with information protection laws, as it happened in the USA for HIPPA, in order to facilitate access to telemedicine services [5]. However, the privacy issue 
remains a compelling one.

\section{Telepsychiatry in the treatment of psychiatric disorders}

The evidence supporting the use of TP in the treatment of depressive disorders is robust [15]. A large number of systematic reviews and meta-analyses have examined technology-based interventions for depression, but only few of those have attempted to synthesize the data with the purpose of determining the admissibility and influencing factors of the implementation of TP [16].

There are different forms of internet-delivered treatments for depression. One approach, based on bibliotherapy and the therapist's guidance via email or phone, uses primarily text-based materials. There are also short treatments that are not generally delivered with guidance and do not usually target people with clinical depression; although they can reach more people at a lower cost, they do so with a presumably diminished effect [17]. Globally, it seems that computerized treatments, administered with the support of a therapist, are much more effective than the unsupported ones, and that is confirmed in many open studies [18].

Patients with depressive disorders have been shown to benefit from TP, as reported by several studies in which patients' symptoms improved more in the telemental health group than in the traditional setting ones. A 2015 study evidenced how patients taking antidepressant in a high-intensity telemedicine-based collaborative care model reported less drug-related side effects than the low- intensity practice-based collaborative care patients [19].

Evidence from several studies has underlined that the use of TP for delivering mental healthcare services can improve symptoms of depression among older adults. The effects are the reduction of depression symptoms and the improvement of comorbid health conditions. A 2013 review analyzed the combination of TP with mobile apps targeting depression, anxiety disorders and substance abuse, reporting similar results [20]. Stanmore et al., in a meta-analytic review, showed that TP improves not only the patient's global cognition score, but also his/her cognitive functioning, which is often affected in depressive disorders [21].

In 2019 Pruitt and colleagues assessed patients' satisfaction in a group of military personnel with depression , who were included in a randomized clinical trial that utilized a type of psychotherapy known as Behavioral Activation Therapy for Depression (BATD); this was delivered in-office and at home by videoconferencing [22]. They discovered that patients' satisfaction was very high, with no significant difference between the two modalities, which is indicative of a comparable quality of care, and a satisfying level of therapeutic alliance between the patients and the clinicians. The two treatment modalities have diverse strengths and weaknesses: in-person treatments offer a richer communication environment, both verbal and non-verbal, when compared to the other; yet the in-home treatments may grant greater comfort because of the subjects' ability to relax in a familiar setting. This balance between positive and negative aspects of the two modalities may explain the analogy of patients' satisfaction observed in Pruitt's study. Contrary to popular belief, higher levels of endof-treatment satisfaction for in-person care were detected to be mostly associated with younger age patients. Conversely, the satisfaction for in-home care was more frequently associated with older and symptomatic individuals. Plausible explanations for such results include stigma, symptom severity and convenience. Younger patients, or those with less debilitating symptoms, may be more satisfied with traditional care treatments, while the elderly, or more symptomatic patients, may prefer a home-based care setting [22].

It has been proposed that telemental health strategies could be a useful resource in the treatment of patients with Obsessive Compulsive Disorder (OCD). With this kind of patients several barriers to treatment, such as logistic and financial barriers, as well as shame and fear of stigmatization or discrimination [23], could lead to the customary long delay from the onset of the disorder to the first treatment delivery [24]; telemental health therapies have the potential to overcome these barriers [25]. In their extensive review [12] Herbst et al. highlighted that a lot of telemental health treatments have been used for OCD: computer programs, Computer-Aided Vicarious Exposure (CAVE), Behaviour Therapy-Steps (BT-Steps), metacognitive training, meridian tapping, online Cognitive Behavioural Therapy (CBT) based group interventions, Telephone-administered CBT, Videoconference-based CBT. All these approaches were based on the CBT principles. Therapists' level of involvement ranged from direct contact through video or phone, to completely 
automated computer programs with no direct involvement. All telemental health interventions resulted in a decrease in OCD symptoms for active participants. They observed that the most efficient studies included contact with the therapist (higher effect size and lower dropout rate), although approaches that did not include visual or auditory therapist's contact could reduce the inhibitory effect of shame and self-stigmatisation, hence improving the treatment adherence with some types of patients, such as the ones with aggressive or sexual obsessions. It has been suggested that telemental health treatments for OCD could be useful in the context of a diversified care approach offering all patients a low-threshold self-treatment at first, to then move up to the face-to-face treatment, only when necessary [26] [27].

Bolton \& Dorstyn [28] analyzed the role of tele-psychological services in the management of Post-traumatic Stress Disorder (PTSD), a complex syndrome characterized by the re-experiencing of a traumatic event, persistent avoidance and hyperarousal [29]. In their meta-analysis the authors evaluated studies using either a videoconferencing model only, or a hybrid design, which included the internet as the primary treatment modality in combination with real-time or asynchronous communication with a therapist. All interventions were based on a cognitive behavioral framework and were time limited, with an average duration of three months. They determined that there was a short-term effectiveness of the therapist-assisted internet programs, with a large reduction in the severity of depression, anxiety symptoms and fear reactions, and an immediate improvement in suicidal ideation, social functioning and insomnia. Video-based interventions produced short-term improvements on affective symptoms, even though peers who received face- to-face therapy showed greater treatment progress. A 1 to 6 months follow-up among those who accessed internet programs revealed an increased risk of alcohol consumption over time, but also a decline in cognitive and behavioural symptoms of PTSD and depression. The downside was in the difficulty with online cognitive restructuring tasks and the preference for other media tools (i.e. mobile application) as a supplement to internet-based guided self-help. In a recent Randomized Controlled Trial Haghnia et al. [30] directly compared the efficacy of TP and face-to-face consultation in PTSD treatments in a population of 60 Iranian War Veterans. Patients were divided into an experimental and a control group; both groups were treated through face-to-face consultations for the first 3 sessions. Six follow-up sessions were held remotely, using the Imo mobile application and Skype with the experimental group, and face-to-face with the control group. The researchers observed that, compared with the face-to-face method, TP increased access to psychiatric care, reduced follow-up treatment costs and patients' waiting time, and increased patients' satisfaction and physician-patient communication.

The use of TP has been tested in patients with Schizophrenia as well. In their review, Kasckow et al. [31] summarized many studies involving telephone-based, internet-based and videoconference-based interventions and concluded that TP approaches were feasible with this kind of patients and that they could improve treatment outcomes. TP may help to engage these patients, to improve their medication adherence rates, to detect the exacerbation of psychotic symptoms and to prevent hospitalizations; nonetheless, only a strong patient-clinician engagement seems to guarantee an improvement in clinical and social functioning and an adequate relapse prevention [32]. Patients with Schizophrenia exhibited high satisfaction levels when using telepsychiatry approaches; in fact, many patients reported feeling more comfortable using TP methods to connect with their psychiatrist and, additionally, anonymous web-based interventions may help them reduce stigmatization feelings.

\section{Psychotherapy via telepsychiatry}

Psychotherapy is now the front line, gold standard treatment for common mental health disorders in evidencebased medicine [33]. Yet, due to barriers including access, cost and stigma, estimates suggest that up to two-thirds of individuals do not access or receive evidence-based care [34]. TP could help overcome those barriers.

Herbst et al. [25] showed how, in a population of 34 patients, an Internet-based, therapist-guided CBT with Exposure and Response Prevention led to significant reductions in the main symptoms of OCD; they also observed that this improvement remained stable throughout the 6-month follow-up period after treatment. The i-CBT did not inhibit a solid patient-therapist working alliance; in fact, a strong and stable patient- 
therapist relation was established [35]. Matsumoto et al. [36] examined the feasibility of videoconferencedelivered CBT in 30 adult patients with mild to severe OCD, Panic Disorder (PD) and Social Anxiety Disorder (SAD). They determined that there was a significant reduction in symptoms for each group, with a remission rate of $40 \%$ for OCD, $50 \%$ for $\mathrm{PD}$ and $22 \%$ for SAD. The therapeutic alliance significantly improved throughout the treatment and its scores were comparable to those of in-person CBT. The dropout rate was very low (3\%), since $97 \%$ of patients completed the videoconference-delivered CBT treatment. Interestingly, $83 \%$ of the participants affirmed to prefer videoconference-delivered CBT to face-to-face CBT.

\section{New technologies in mental health care}

Additionally to tele-health services, in recent years digital technologies have found other numerous and relevant declinations. Firstly, the growing mediatic attention towards psychiatric disorders and mental health problems has led to the development of specifically dedicated smartphone applications. Through a search on dedicated platforms conducted in 2019, 1435 mobile apps were identified, 449 of which addressing anxiety problems, 450 addressing Depression, 282 addressing Schizophrenia, 124 addressing self-harm and 140 addressing Substance Use Disorders [37]. This global mobile-Health apps market, which is dominated by Apple's App Store and Google Play Store, is characterized by an annual growth rate of circa 38,3\% starting in 2019 and generating an estimated US 111,1 billions by 2025. Examples of mobile apps' potentialities are the capacity to monitor and track health parameters, tobacco smoke and alcohol consumption, as well as the trend of symptoms, sleep and stress levels. They can also provide electronic reminders for the correct management of pharmacotherapy and can help patients to keep in touch with health care providers.

Secondly, the applications of Virtual Reality (VR) are very promising for the mental health field. VR is defined as a computer-generated simulation, a set of computer generated images and sounds, representing a place or situation with which the person can interact utilizing special electronic equipment [38]. Immersion in VR allows the patient to savor emotions he has already experienced in the real world, yet also to face dysfunctional behaviors and traumatic situations in a safe setting. It allows each individual to act as the protagonist and to regain control of his actions, to feel as the active builder of his own experience and, over time, of the changes he brings into his own life [39]. The best applications of VR are found in PTSD, specific phobias, body-image disorders, such as nervous anorexia, and autism spectrum disorders.

Finally, today psychiatrists have the unprecedented opportunity to benefit from the use of Artificial Intelligence (AI) and, more specifically, of Machine Learning. This technique allows the clinicians to analyze huge amounts of data with the aim of producing a biologically founded re-classification of Major Psychiatric Disorders. Increasing evidence suggests that the sub-classifications obtained from Machine Learning data analysis have better predictive power of treatment outcome than the traditional DSM/ICD models. In a new era of evidence-based psychiatry these objectively measurable transdiagnostic endophenotypes will allow the clinicians to make an early diagnosis, to individualize treatment and to make therapeutic adjustments to reduce disease relapses that are tailored for each individual. This has been called Computational Psychiatry [40].

\section{Discussion}

TP is a new and growing field with excellent prospects and many promising clinical applications. It has the potential to geographically redistribute specific mental health services.

Telemental health services are particularly feasible and appropriate for the support of patients, family members, and health-care providers during the COVID-19 pandemic. Treatment protocols for people with COVID-19 should approach both the physical and psychological uneasiness of patients and health-care providers. Psychological support may reduce the burden of comorbid mental health conditions and ensure the wellbeing of those affected. This highlights the actual role of TP.

TP is globally underused: in 2009, only about $2 \%$ of psychiatrists in the United States had used TP [41]. A recent article examining a large population of subjects from 2005 to 2027 concluded that, although telemedicine care had substantially increased during that time, its use was still not widespread in 2017 
[42]. In addition, in 2014, the same 100 clinicians accounted for more than half of all tele-mental health visits that year [43], suggesting that TP practice had been undertaken by very few, selected clinicians. The pandemic crisis can contribute to spread, among numerous mental health professionals, the knowledge of the possibilities offered by the digital era.

However, more studies are needed to ensure a broader and more reliable validation of TP, in terms of diagnostic reliability and therapeutic efficiency in the short and long term. Indeed, the development and the evaluation of standard protocols and more detailed practice guidelines are essential to a broader implementation of such tools. Likewise, focused and continuous trainings of clinicians are required to increase the awareness of the benefits and risks of TP. In relation to this, and considering the present pandemic, formally teaching TP in medical schools, residency trainings and continuing medical education programs should be encouraged. Among other benefits, this may be a way to help cultural acceptance of TP both among patients and medical staff.

The COVID-19 pandemic situation shows us how vast the amount of untreated mental illnesses can be, both nationally and internationally; thus, there is an urgent need for a feasible integration of technology with innovative models of mental healthcare. TP is destined to expand in the future: the newest and most exciting frontiers include mobile apps, virtual reality, big data and Artificial Intelligence. Another field in expansion is the so-called "hybrid" model of care, which combines both in-person and technology-driven care, the latter of which can include e-mail, instant messaging, asynchronous telemedicine and much more. An important issue is to identify which patients are most appropriate for which models of TP. Mental health professionals have a strategic role to help ensure that these technologies respect the therapeutic relationship and remain rigorous in their scientific foundation.

\section{References}

[1] Zhou X, Snoswell MMHCL, Harding LE, et al. The Role of Telehealth in Reducing the Mental Health Burden from COVID-19. 2020;26:26-28.

[2] Corruble E. A Viewpoint From Paris on the COVID-19 Pandemic: A Necessary Turn to Telepsychiatry. J Clin Psychiatry. 2020;2020.

[3] WHO. A health telematics policy in support of WHO's Health-For-All strategy for global health development: report of the WHO group consultation on health telematics. 1997;

[4] Hilt RJ. Telemedicine for Child Collaborative or Integrated Care. Child Adolesc. Psychiatr. Clin. N. Am. 2017;26:637-645.

[5] Wright JH, Caudill R. Remote Treatment Delivery in Response to the COVID-19 Pandemic. Psychother. Psychosom. 2020;40202:1-3.

[6] Chan S, Li L, Torous J, et al. Review of Use of Asynchronous Technologies Incorporated in Mental Health Care. 2018;

[7] Cowan KE, McKean AJ, Gentry MT, et al. Barriers to Use of Telepsychiatry: Clinicians as Gatekeepers. Mayo Clin. Proc. 2019;94:2510-2523.

[8] Richardson LK. Current Directions in Videoconferencing Tele-Mental Health Research. 2009;323-338.

[9] Hilty DM, Sunderji N, Suo S, et al. International Review of Psychiatry Telepsychiatry and other technologies for integrated care : evidence base, best practice models and competencies. Int. Rev. Psychiatry. 2019;0:1-18.

[10] May C, Gask L, Atkinson T, et al. Resisting and promoting new technologies in clinical practice : the case of telepsychiatry. 2001;52:1889-1901.

[11] Abrams J, Sossong S, Schwamm LH, et al. Practical Issues in Delivery of Clinician-to-Patient Telemental Health in an Academic Medical Center. 2017;135-145. 
[12] Herbst N, Voderholzer U, Stelzer N, et al. The potential of telemental health applications for obsessivecompulsive disorder. Clin. Psychol. Rev. 2012;32:454-466.

[13] Mclaren P, Baht CJ, Summerfield AB, et al. An evaluation of the use of interactive television in an acute psychiatric service. J Telemed Telecare. 1995;79-85.

[14] Barton PL, Brega AG, Devore PA, et al. Specialist Physicians' Knowledge and Beliefs about Telemedicine: A Comparison of Users and Nonusers of the Technology. Telemed. e-health. 2007;13.

[15] Andersson. Internet-Based and Other Computerized Psychological Treatments for Internet-Based and Other Computerized Psychological Treatments for Adult Depression : A Meta-Analysis. 2009;

[16] Harerimana B, Forchuk C, Regan TO. The use of technology for mental healthcare delivery among older adults with depressive symptoms : A systematic literature review. 2019;657-670.

[17] Spek. Internet-based cognitive behaviour therapy for symptoms of depression and anxiety : a metaanalysis. Psychol Med. 2007;319-328.

[18] Christensen H, Griffiths K, Groves C, et al. Free range users and one hit wonders : community users of an Internet-based cognitive behaviour therapy program. Aust N Z J Psychiatry. 2006;

[19] Chan S, Parish M, Yellowlees P, et al. Telepsychiatry Today. Curr Psychiatry Rep. 2015;1-9.

[20] Donker, T., Petrie, K., Proudfoot, J., Clarke, J., Birch, M. R. \& Christensen H. Smartphones for smarter delivery of mental health programs: A systematic review. J. Med. Internet Res. 2013;

[21] Stanmore E, Stubbs B, Vancampfort D, et al. The effect of active video games on cognitive functioning in clinical and non-clinical populations : A meta-analysis of randomized controlled trials. Neurosci. Biobehav. Rev. 2017;78:34-43.

[22] Pruitt LD, Vuletic S, Smolenski DJ, et al. Predicting post treatment client satisfaction between behavioural activation for depression delivered either in-person or via home-based telehealth. J Telemed Telecare. 2019;25:460-467.

[23] Marques L, LeBlanc NJ, Wegarden HM, et al. Barriers to treatment and service utilization in an internet sample of individuals with obsessive-compulsive symptoms. Depress. Anxiety. 2010;27:470-475.

[24] Pinto A, Mancebo MC, Eisen JL, et al. The Brown Longitudinal Obsessive Compulsive Study: Clinical features and symptoms of the sample at intake. J. Clin. Psychiatry. 2006;67:703-711.

[25] Herbst N, Voderholzer U, Thiel N, et al. No talking, just writing! efficacy of an internet-based cognitive behavioral therapy with exposure and response prevention in obsessive compulsive disorder. Psychother. Psychosom. 2014;83:165-175.

[26] Mataix-Cols D, Marks IM. Self-help with minimal therapist contact for obsessive-compulsive disorder: A review. Eur. Psychiatry. 2006;21:75-80.

[27] Tolin DF, Diefenbach GJ, Gilliam CM. Stepped care versus standard cognitive-behavioral therapy for obsessive-compulsive disorder: A preliminary study of efficacy and costs. Depress. Anxiety. 2011;28:314-323.

[28] Bolton AJ, Dorstyn DS. Telepsychology for Posttraumatic Stress Disorder: A systematic review. J. Telemed. Telecare. 2015;21:254-267.

[29] Martinotti G, Sepede G, Brunetti M, et al. BDNF concentration and impulsiveness level in posttraumatic stress disorder. Psychiatry Res. 2015;229:814-818.

[30] Haghnia Y, Samad-Soltani T, Yousefi M, et al. Telepsychiatry- based care for the treatment follow-up of iranian war veterans with post- traumatic stress disorder: A randomized controlled trial. Iran. J. Med. Sci. 2019;44:291-298. 
[31] Kasckow J et al. telepsychiatry in the assessment and treatment of schizophrenia. Clin. Schizophr. Relat Psychoses. 2014;

[32] Krzystanek M, Krysta K, Ska K. Treatment Compliance in the Long-Term Paranoid Schizophrenia Telemedicine Study. 2017;84-87.

[33] NICE. Depression in Adults: Treatment and Management. 2019;

[34] Lake J. Urgent Need for Improved Mental Health Care and a More Collaborative Model of Care. Perm J. $2017 ; 17-24$.

[35] Herbst N, Franzen G, Voderholzer U, et al. Working Alliance in Internet-Based Cognitive-Behavioral Therapy for Obsessive-Compulsive Disorder. Psychother. Psychosom. 2016;85:117-118.

[36] Matsumoto K, Sutoh C, Asano K, et al. Internet-based cognitive behavioral therapy with real-time therapist support via videoconference for patients with obsessive-compulsive disorder, panic disorder, and social anxiety disorder: Pilot single-arm trial. J. Med. Internet Res. 2018;20.

[37] Larsen ME, Huckvale K, Nicholas J, et al. Using science to sell apps : Evaluation of mental health app store quality claims. Digit. Med. 2019;

[38] Park MJ, Kim DJ, Lee U, et al. A Literature Overview of Virtual Reality ( VR ) in Treatment of Psychiatric Disorders : Recent Advances and Limitations. Front Psychiatry. 2019;10:1-9.

[39] Riva G. Transforming experience : The Potential of Augmented Reality and virtual Reality for enhancing Personal and Clinical Change. Front Psychiatry. 2016;7:1-14.

[40] Bzdok D, Meyer-lindenberg A. Review Machine Learning for Precision Psychiatry : Opportunities and Challenges. Biol. Psychiatry Cogn. Neurosci. Neuroimaging. 2018;3:223-230.

[41] Yellowlees P, Nafiz N. The Psychiatrist-Patient Relationship of the Future : Anytime, Anywhere ? Harv Rev Psychiatry. 2010;96-103.

[42] Barnett. Trends in Telemedicine Use in a Large Commercially Insured Population, 2005-2017. JAMA. 2018;320:2017-2019.

[43] Mehrotra A, Huskamp HA, Souza J, et al. Rapid growth in mental health telemedicine use among rural medicare beneficiaries, wide variation across States. Heal. Aff. 2017; 